Left subhereditary supernilpotent radical classes

D. Isabel C. Mendes and Sodnomkhorloo Tumurbat 
Mathematical Notes, Miskolc, Vol. 2., No. 1., (2000), pp. 75-80

\title{
LEFT SUBHEREDITARY SUPERNILPOTENT RADICAL CLASSES
}

\author{
D. Isabel C. Mendes \\ Universidade da Beira Interior \\ Covilhã, Portugal \\ dmendes@noe.ubi.pt \\ SODNOMKHORLOO TUMURBAT \\ University of Mongolia, P.O.Box 75 \\ Ulaan Baatar 20, Mongolia \\ tumur@www.com
}

[Received March 17, 2001]

\begin{abstract}
Left) subhereditary radicals were introduced in [5]. Here, we continue to study such radicals and give some characterizations of supernilpotent subhereditary radicals.

Mathematical Subject Classification: $16 \mathrm{~N} 80$

Keywords: Subhereditary and supernilpotent radical, weakly special class of rings
\end{abstract}

\section{Preliminaries}

In this note rings are associative, not necessarily with unit elements. As usual, $I \triangleleft A$ and $L \triangleleft_{\ell} A$ denote, respectively, that $I$ is an ideal and $L$ is a left ideal in the ring $A$.

Let us recall that a (Kurosh-Amitsur) radical $\gamma$ is a class of rings, which is closed under homomorphisms, extensions ( $I$ and $A / I$ in $\gamma$ imply $A$ in $\gamma$ ), and has the inductive property (if $I_{1} \subseteq \cdots \subseteq I_{\lambda} \cdots$ is a chain of ideals of the ring $A=\cup I_{\lambda}$ and each $I_{\lambda}$ is in $\gamma$, then $A$ is in $\gamma$ ). Every ring $A$ contains a unique largest $\gamma$-ideal (i.e. an ideal which is in $\gamma), \gamma(A)$, which is called the $\gamma$-radical of $A$.

A radical $\gamma$ determines its semisimple class

$$
S \gamma=\{A \mid \gamma(A)=0\}
$$

which is always a hereditary class $(I \triangleleft A \in S \gamma$ implies $I \in S \gamma)$.

A class $\mathcal{M}$ of rings is said to be regular, if every nonzero ideal of a ring in $\mathcal{M}$ has a nonzero homomorphic image in $\mathcal{M}$. Starting from a regular (in particular, hereditary) class $\mathcal{M}$ of rings, the upper radical operator $\mathcal{U}$ yields a radical class:

$$
\mathcal{U M}=\{A \mid A \text { has no nonzero homomorphic image in } \mathcal{M}\} .
$$

Remember that the Baer radical $\beta$ is the upper radical determined by all prime rings. A radical $\gamma$ containing all nilpotent rings is called a hypernilpotent radical. A 
radical $\gamma$ is said to be supernilpotent if it is hereditary and hypernilpotent. A left ideal $L$ of a ring $A$ is said to be essential if $I \cap L \neq 0$ for every $0 \neq I \triangleleft A$ and is denoted by $L \triangleleft_{\ell}^{\bullet} A$. A radical $\gamma$ is said to be (left) subhereditary if

$$
0 \neq L \triangleleft_{\ell} A \in \gamma \text { implies } \gamma(L) \neq 0,
$$

or equivalently

$$
0 \neq L \triangleleft_{\ell}^{\bullet} A \text { and } \gamma(L)=0 \text { imply } \gamma(A)=0
$$

(see [5]).

A class $\mathcal{M}$ of prime (semi-prime) rings is special (weakly special) if it is hereditary and closed under essential extensions (if $I \in \mathcal{M}$ is an essential ideal of a ring $A$, then also $A \in \mathcal{M}$ ).

The upper radical $\mathcal{U M}$ of a special (weakly special) class $\mathcal{M}$ of rings is called a special radical (weakly special radical).

\section{Subhereditary and hypernilpotent radicals}

PROPOSITION 1. If $\gamma$ is a subhereditary radical and $A$ is any semiprime ring, then every $\gamma$-semisimple left ideal $L$ of $A$ can be extended to a unique largest ideal $I$ of $A$ such that $\gamma(A) \cap I=0$.

Proof. First, we shall prove that $\gamma(A) \cap L=0$. Suppose that this is not the case. Since $\gamma$ is subhereditary and

$$
\gamma(A) \cap L \triangleleft_{\ell} \gamma(A) \in \gamma,
$$

it follows that $\gamma(\gamma(A) \cap L) \neq 0$. By $\gamma(A) \cap L \triangleleft L$, also

$$
0 \neq \gamma(\gamma(A) \cap L) \subseteq \gamma(L),
$$

contradicting the assumption on $L$. Thus $\gamma(A) \cap L=0$ holds, implying $\gamma(A) L=0$. Define

$$
K=\sum\left(L \triangleleft_{\ell} A / 0 \neq L \in S \gamma\right) \triangleleft_{\ell} A .
$$

We also have $\gamma(A) K=0$. The ideal $I$ of $A$ generated by $K$ is $I=K+K A$. Now $\gamma(A) \cap I \triangleleft A$ and

$$
(\gamma(A) \cap I)^{2} \subseteq \gamma(A) I=\gamma(A)(K+K A)=0 .
$$

Since $A$ is semiprime, we conclude that $\gamma(A) \cap I=0$.

PROPOSITION 2. Let $\gamma$ be a hypernilpotent radical. If in every semiprime ring $A$ each $\gamma$-semisimple left ideal $L$ can be extended to a unique largest ideal I of $A$ such that $\gamma(A) \cap I=0$, then $\gamma$ is subhereditary.

Proof. Let $0 \neq L \triangleleft_{\ell}^{\bullet} A$ and $\gamma(L)=0$. We prove the validity of condition $(\diamond)$. If the ring $A$ is semiprime, then, by the assumption, there exists an ideal $I$ of $A$ such that 
$L \subseteq I$ and $\gamma(A) \cap I=0$. Hence also $\gamma(A) \cap L=0$. Since $L$ is an essential left ideal of $A$, it follows that $\gamma(A)=0$.

If $A$ is not semiprime, then $\beta(A) \neq 0$. Clearly $\beta(A) \cap L \triangleleft L$ and $\beta(A) \cap L \triangleleft \ell \beta(A)$. Since $L \triangleleft_{\ell}^{\bullet} A$, we have by the left hereditariness of $\beta$ that

$$
0 \neq \beta(A) \cap L \subseteq \beta(L) .
$$

Since $\gamma$ is hypernilpotent, also $\gamma(L) \neq 0$, contradicting $\gamma(L)=0$.

COROLLARY 3. Let $\gamma$ be a subhereditary radical. If a semiprime ring $A$ has a nonzero $\gamma$-semisimple left ideal $L$, then either $\gamma(A)=0$ or $A$ is not a subdirectly irreducible ring.

THEOREM 4. Let $\gamma$ be a hypernilpotent radical. Then the following are equivalent:

i) $\gamma$ is subhereditary.

ii) If $A$ is a semiprime ring, $L \triangleleft_{\ell} A$ and $\gamma(A) L \neq 0$, then $\gamma(L) \neq 0$.

iii) In every semiprime ring $A$ each $\gamma$-semisimple left ideal $L$ can be extended to a unique largest ideal $I$ of $A$ such that $\gamma(A) \cap I=0$.

Proof. The equivalence i) $\Leftrightarrow$ iii) follows from Propositions 1 and 2.

i) $\Rightarrow$ ii): Let $\gamma$ be a subhereditary radical, $A$ a semiprime ring and $L \triangleleft_{\ell} A$, such that $\gamma(A) L \neq 0$. Clearly $\gamma(A) L \triangleleft L$ and $\gamma(A) L \triangleleft_{\ell} \gamma(A)$. Since $\gamma$ is subhereditary, $0 \neq \gamma(\gamma(A) L) \triangleleft L$ and so $\gamma(L) \neq 0$.

ii) $\Rightarrow \mathrm{i})$ : Let $0 \neq L \triangleleft_{\ell} \gamma(A)$. Suppose that $\gamma(L)=0$. Let $I \triangleleft \gamma(A)$ such that $I$ is maximal with respect to $I \cap L=0$. Then $\gamma(A) / I$ is a semiprime ring. Indeed, if not, then $\beta(\gamma(A) / I) \neq 0$ and so $\beta(\gamma(A) / I) \cap(L+I) / I \neq 0$. Clearly

$$
L=L / L \cap I \cong(L+I) / I=\bar{L} .
$$

Hence $0 \neq \beta(\bar{L})$ and so $\beta(L) \neq 0$.

Now we get $(\gamma(A) / I) \bar{L} \neq 0$ and so $\gamma(\bar{L}) \neq 0$. Since $\bar{L} \cong L$, we have $\gamma(L) \neq 0$, a contradiction.

For a subset $S$ of a ring $A$, we denote the left (right) annihilator of $S$ in $A$ by $\ell(A, S)$, (and $r(A, S)$, respectively). Further, let $P$ denote the class of all prime rings.

THEOREM 5. For a special class $\mathcal{M}$, the following conditions are equivalent:

i) $\gamma=\mathcal{U M}$ is subhereditary.

ii) If $A$ is a semiprime ring with $\gamma(A) \neq 0$ and $L \triangleleft_{\ell}^{\bullet} A$, then $\gamma(L) L \neq 0$.

iii) Let $B=\sum\left(B_{\lambda} \in \gamma \cap P \mid\right.$ there exists $0 \neq L_{\lambda} \triangleleft_{\ell} B_{\lambda}$ with $\left.L_{\lambda} / r\left(L_{\lambda}, L_{\lambda}\right) \in \mathcal{M}\right)$. If $L \triangleleft_{\ell} B$ and $L \in \mathcal{S} \gamma$, then $B \in \mathcal{S} \gamma$.

Proof. i $\Rightarrow$ ii). Suppose that $\gamma$ is subhereditary, $L \triangleleft_{\ell}^{\bullet} A$ and $\gamma(A) \neq 0$. Since $L \triangleleft_{\ell}^{\bullet} A$, $\gamma(A) \cap L \neq 0$. Clearly $\gamma(A) \cap L \triangleleft L$ and $\gamma(A) \cap L \triangleleft_{\ell} \gamma(A)$. Since $\gamma$ is subhereditary,

$$
0 \neq \gamma(\gamma(A) \cap L) \subseteq \gamma(L),
$$


it follows $\gamma(L) \neq 0$. If $\gamma(L) L=0$, then $\ell(A, L) \neq 0$. Since $L \triangleleft_{\ell}^{\bullet} A$,

$$
0 \neq \ell(A, L) \cap L \triangleleft_{\ell} A \text { and so }(\ell(A, L) \cap L)^{2}=0 .
$$

Therefore $\beta(A) \neq 0$, contradicting $A$ is semiprime. Thus $\gamma(L) L \neq 0$.

ii) $\Rightarrow$ iii) Suppose $L \triangleleft_{\ell}^{\bullet} B$ and $L \in S \gamma$. Since $B_{\lambda}$ is prime for each index $\lambda, B$ is semiprime. If $\gamma(B) \neq 0$, then $\gamma(L) L \neq 0$, and so $\gamma(L) \neq 0$, a contradiction.

iii) $\Rightarrow$ i). Let $0 \neq L \triangleleft_{\ell} A$, where $A \in \gamma$. Suppose that $L \in S \gamma$. Without loss of generality we can choose $L \triangleleft_{\ell}^{\bullet} A$. Since $\gamma$ is a special radical, $L$ is a subdirect sum of prime rings $0 \neq L / I_{\lambda} \in \mathcal{M}$, where $\cap I_{\lambda}=0$. By Lemma 3 [1], there exist prime ideals $A_{\lambda}$ of $A$, for each $\lambda$ such that

$$
I_{\lambda}^{2} \subseteq L \cap A_{\lambda} \subseteq I_{\lambda}
$$

Put $B_{\lambda}=A / A_{\lambda}$ and $L_{\lambda}=\left(L+A_{\lambda}\right) / A_{\lambda}$. Therefore $\gamma\left(L_{\lambda}\right)=\gamma\left(\left(L+A_{\lambda}\right) / A_{\lambda}\right) \cong$ $\gamma\left(L / L \cap A_{\lambda}\right)=I_{\lambda} / L \cap A_{\lambda} \in \beta$, where $\beta$ is Baer radical. It is easy to see $L_{\lambda} \gamma\left(L_{\lambda}\right)=0$ and so $\gamma\left(L_{\lambda}\right)=r\left(L_{\lambda}, L_{\lambda}\right)$. We show that $\cap A_{\lambda}=0$. If $0 \neq \cap A_{\lambda}$, then $0 \neq L \cap\left(\cap A_{\lambda}\right)=$ $\cap\left(L \cap A_{\lambda}\right) \subseteq \cap I_{\lambda}=0$, a contradiction. Hence $A=\sum_{\text {subdirect sum }} B_{\lambda}$, and clearly $B_{\lambda} \in \gamma$. Since $L \in S \gamma$, by assumption $A \in S \gamma \cap \gamma=0$, contradicting $0 \neq L$.

DEFINITION 6. A class $\mathcal{M}$ of rings is called a left special (left weakly special) class of rings if the following conditions are satisfied:

i) $\mathcal{M}$ consists of prime (semiprime) rings.

ii) $\mathcal{M}$ is hereditary.

iii) If $A$ is a semiprime ring, $0 \neq L \triangleleft_{\ell}^{\bullet} A$ and $L \in \mathcal{M}$, then $A \in \mathcal{M}$.

Let $P$ denote the class of all prime rings.

THEOREM 7. i) A hypernilpotent radical $\gamma$ is subhereditary if and only if $S \gamma$ is a left weakly special class of rings.

ii) A hypernilpotent radical $\gamma$ is subhereditary and special if and only if $S \gamma$ is left weakly special and $\gamma=\mathcal{U}(S \gamma \cap P)$.

Proof. i) Let $\gamma$ be a subhereditary radical. Then by $(\diamond)$, if $L \in S \gamma$ and $L \triangleleft_{\ell}^{\bullet} A$, then $\gamma(A)=0$, and so $A \in S \gamma$. Since $\gamma$ is hypernilpotent, $S \gamma$ consists of semiprime rings, and $S \gamma$ is hereditary.

Suppose $S \gamma$ is a left weakly special class. If $L \in S \gamma$ and $L \triangleleft_{\ell}^{\bullet} A$ then $A \in S \gamma$. Again by $(\diamond), \gamma$ is subhereditary. class.

ii) $\Rightarrow$ : Since $\gamma$ is a special, clearly $\gamma=\mathcal{U}(S \gamma \cap P)$. By i) $S \gamma$ is a left weakly special

$\Leftarrow$ : Again by i) $\gamma$ is a subhereditary. Since $S \gamma$ is a left weakly special class, $S \gamma \cap P$ is a special class and so $\gamma$ is special.

LEMMA 8. Let $\left\{H_{i}: i \in \Lambda\right\}$ be a family of subhereditary and weakly special radicals. 
Then $\mathcal{L}\left(\cup H_{i}\right)$, the lower radical class determined by $\cup H_{i}$, is also a subhereditary and weakly special radical class.

Proof. We know that $\mathcal{L}\left(\cup H_{i}\right)$ is a weakly special radical. By [5, Proposition 2.2] $\mathcal{L}\left(\cup H_{i}\right)$ is subhereditary.

In a similar way to ([3], Theorem 4) we can use Lemma 8 to prove the following theorem:

THEOREM 9. A radical class $\mathcal{H}$ contains the largest subhereditary special radical class if and only if it contains the class $\mathcal{Z}$ of all zero rings.

The above theorem and ([2], Proposition 2) give rise to the following:

COROLLARY 10. A radical class $\mathcal{H}$ contains the largest subhereditary radical class if and only if $\mathcal{H}$ contains the largest weakly special radical class.

The following theorem can be proved in a similar way to ([3], Theorem 5).

THEOREM 11. The following conditions are equivalent for a class $\mathcal{M}$ of rings:

(i) $\mathcal{U M}$ is a radical class containing a subhereditary special radical class.

(ii) $\mathcal{U M}$ is a radical class containing a subhereditary weakly special radical class.

(iii) (a) Every $0 \neq A \in \mathcal{M}$ has some non-zero image in $\mathcal{S U M}$.

(b) $\mathcal{S U M} \subseteq \mathcal{E}$ where $\mathcal{E}$ denotes the class of all semi-prime rings.

COROLLARY 12. (see [4], Corollary 1) The following conditions are equivalent for an arbitrary class $\mathcal{M}$ of rings:

(i) $\mathcal{U M}$ is a radical class containing a subhereditary special radical class.

(ii) $\mathcal{U M}$ is a radical class containing a subhereditary weakly special radical class.

(iii) $\mathcal{U M}$ is a radical class containing a special left strong radical class.

(iv) $\mathcal{U M}$ is a radical class containing a weakly special left strong radical class.

(v) $\mathcal{U M}$ is a radical class containing a hereditary left special radical class.

(vi) $\mathcal{U M}$ is a radical class containing a hereditary left weakly special radical class.

(vii) $\mathcal{U M}$ is a radical class containing a left special radical class.

(viii) $\mathcal{U M}$ is a radical class containing a weakly special radical class.

(ix) $\mathcal{U M}$ is a hypernilpotent radical class.

For a hereditary class $\mathcal{M}$ of semiprime rings, let us define

$$
\mathcal{M}_{k}=\{A \mid A \text { has an essential left ideal in } \mathcal{S U M}\} .
$$

We terminate this paper with the following theorem: 
THEOREM 13. Let $\mathcal{M}$ be a hereditary class of semiprime rings. Then the following statements are equivalent:

(i) $\mathcal{U M}$ is a left subhereditary radical class.

(ii) $\mathcal{U M}=\mathcal{U M}_{k}$.

(iii) $\mathcal{U M} \cap \mathcal{M}_{k}=0$.

(iv) $\mathcal{M}_{k} \subseteq \mathcal{S U M}$.

(v) Every $0 \neq R \in \mathcal{M}_{k}$ has some non-zero image in $\mathcal{S U M}$.

(vi) $\mathcal{U M}$ has the intersection property relative to $\mathcal{M}_{k}$; that is, for any ring $R$, the radical $\mathcal{U M}(R)=\cap\left\{I: R / I \in \mathcal{M}_{k}\right\}$.

Proof. First we show that (i) implies (ii). Since $\mathcal{M} \subseteq \mathcal{M}_{k}$, we have that $\mathcal{U} \mathcal{M}_{k} \subseteq \mathcal{U M}$. Now if $R \in \mathcal{M}_{k}$, then $R$ has an essential left ideal in $\mathcal{S U M}$ and, if $\mathcal{U M}$ is left subhereditary, it follows that $R \in \mathcal{S U M}$ and so $\mathcal{U M} \subseteq \mathcal{U} \mathcal{M}_{k}$. To show that (ii) implies (i), suppose that $\mathcal{U M}=\mathcal{U M M}_{k}$. Let $R \in \mathcal{U M}$ and $0 \neq L \triangleleft_{\ell} R$. If $L \in \mathcal{S U M}$ and $I \triangleleft R$ is maximal with respect to $I \cap L=0$, then $L \cong(L+I) / I \triangleleft_{\ell}^{\bullet} R / I$ and, obviously, $R / I \in \mathcal{M}_{k}$. But this is a contradiction with $R \in \mathcal{U} \mathcal{M}=\mathcal{U} \mathcal{M}_{k}$. It is easy to prove that (ii) implies (iii), (iii) implies (iv), (iv) implies (v) and (v) implies (ii). The proofs of (iv) implies (vi) and (vi) implies (v) are analogous to the proofs of (iv) implies (vi) and (vi) implies (v) of ([3], Theorem 6).

Acknowledgement: The authors wish to express their indebtedness and gratitude to Prof. R. Wiegandt for his invaluable advice. The authors gratefully acknowledge the support of the Centre of Mathematics of Universidade da Beira Interior and the Hungarian National Foundation for Scientific Research Grant \# T23434.

\section{REFERENCES}

[1] Beidar, K. I. and Salavová, K.: On the lattice of $N$-radicals, left strong radicals and left hereditary radicals, Acta Math. Hungar. 42, (1983), 81-95.

[2] Leavitt, W. G.: Special closure, $M$-radicals and relative complements, Acta Math. Acad. Sci. Hungar., 28, (1976), 55-67.

[3] Mendes, D. I. C.: Left hereditary supernilpotent radical classes, Acta Math. Hungar., 71, (1996), 123-129.

[4] Mendes, D. I. C.: A note on left strong supernilpotent radical classes, Acta Math. Hungar., 76, (1997), 351-355.

[5] PuczyŁowski, E. R. and Zand, H.: Subhereditary radicals of associative rings, Algebra Colloquium, 6, (1999), 215-223. 